\title{
A tribute to Ernest Beutler
}

rnest Beutler was born in 1928 in Berlin. With the rise of Nazism, Ernie and his family immigrated to Milwaukee, Wisconsin, in 1935. Within a decade, Ernie mastered English, became captivated with medicine and enrolled in an accelerated high school, undergraduate, and medical program, graduating from the University of Chicago with his medical degree in 1950. Following an internship and junior residency in medicine at the University of Chicago, the Korean War found Ernie a first lieutenant in the medical corps, working on the pathogenesis of hemolytic anemia seen in some individuals given primaquine for malaria prophylaxis. These studies, based on Ernie's observations of Heinz bodies and reduced levels of glutathione in affected individuals, established the importance of erythrocyte glucose-6-phosphate dehydrogenase (G6PD) in protection against hemolysis in genetically susceptible individuals.

Following his discharge from the Army, Ernie returned to the University of Chicago to complete his medical residency and was soon appointed assistant professor. There, Ernie's interest in hematological research was encouraged by Leon Jacobson, and Ernie developed productive research projects in iron metabolism and erythrocyte enzymology. In 1959, Ernie was appointed chair of medicine at the City of Hope Medi-

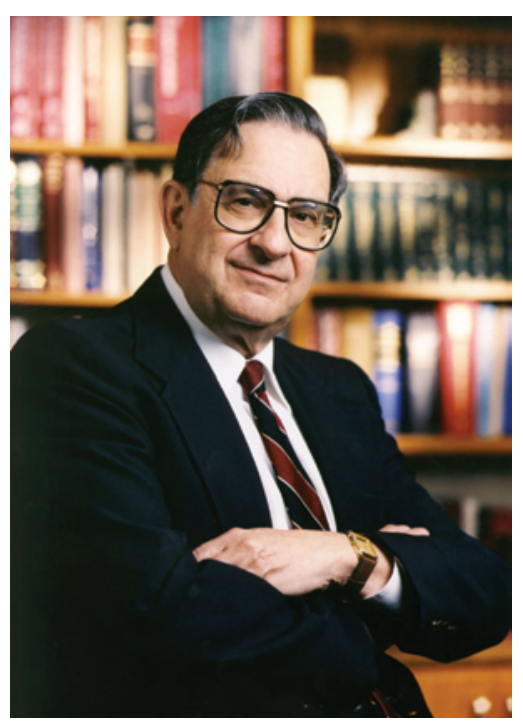

Figure 1

Courtesy of The Scripps Research Institute. cal Center in Duarte, California, where his biomedical interests expanded to genetics. In his 19-year tenure at City of Hope, Ernie penned nearly 300 research publications, including landmark studies that identified the process of random X-chromosome inactivation and established its functional importance (at the same time as Mary Lyon's study). He identified a mutation that causes hereditary galactosemia and purified the enzyme deficient in TaySachs disease. The physician side of Ernie also blossomed; with one of his postdoctoral fellows, Karl Blume, Ernie developed a bone marrow transplant program, establishing allogeneic transplantation as the treatment of choice for patients with acute myelogenous leukemia in first remission.

In 1978, Ernie was appointed chair of the department of molecular and experimental medicine at the Scripps Research Institute in La Jolla, California (Figure 1). At TSRI Ernie's impact continued to grow, both directly from the productivity of his laboratory, and by the collaborative incubator he created for the physician-scientists he helped recruit. Among the stars recruited to TSRI were Bernard Babior, Bruce Beutler, Dennis Carson, Thomas Deuel, Thomas Kipps, Joseph Prchal, and Zaverio Ruggeri.

I first met Ernie in 1990, when he and Bernie Babior attempted to recruit me to TSRI. Ernie showed me "my" cliff-side office with a $180^{\circ}$ view of the Pacific (which I later found out was never filled, but instead was reserved for recruitment purposes!) and demonstrated for me the $\alpha$-test version of Reference Manager, the program he had developed to replace his marginal punch cards and keep track of the more than 500 papers he then cited. Ernie's medical accomplishments also continued, including the development, with Dennis Carson, of cladribine, an effective therapeutic in a number of lymphoproliferative syndromes, especially hairy cell leukemia, where its use leads to a $90 \%$ ten-year survival rate.

In addition to these conventional physician-scientist translations, Ernie displayed a healthy skepticism toward medical dogma. With the cloning of glucocerebrosidase, a recombinant solution to the treatment of Gaucher disease was at hand. Full replacement therapy, recommended by the conventional medical wisdom, dictated the use of $130 \mathrm{U} / \mathrm{kg}$ per month, costing a $70-\mathrm{kg}$ patient approximately $\$ 400,000$ per year. Ernie argued, based on his experimental data, that as little as $10 \%$ of this amount, when administered in divided doses, was sufficient to reverse the pathogenic tissue infiltration, an approach now considered standard of care. Likewise, with the cloning of HFE, a gene highly associated with hereditary hemochromatosis, the literature was soon replete with predictions that individuals homozygous for the $\mathrm{C} 282 \mathrm{Y}$ allele of HFE were certain to develop disease, and appropriate preventative measures should thus be taken. Based on his studies of over 40,000 DNA samples from a large southern California health care organization, Ernie argued that HFE C282Y is necessary but not sufficient for hemochromatosis; once again, Ernie's experimental rigor and common sense prevailed over conventional medical knowledge.

While the productive years of most gifted physician-scientists last two or three decades, Ernie continued to probe and investigate for over 50 years, with the same remarkable energy in his final decade as in his studies of primaquine-induced hemolysis. As an example, about seven years ago, as my tenure as editor of Blood was ending, I wondered whether serving as the chair of a research-intense department of medicine was a reasonable next career challenge. Ernie was 74 years old, so I called my good friend Bernie Babior to inquire whether Ernie might soon retire as chair at TSRI. Once Bernie's laughter died down, he suggested it would take an act of God for Ernie to step down.

After a four-year, heroic battle with lymphoma, Ernie passed away in October. In his illustrious career, Ernie Beutler was elected to the American Society of Clinical Investigation, the Association of American Physicians, the American Academy of Arts and Sciences, the National Academies of Science, and the Institute of Medicine. He will be greatly missed by his family, his many friends, and the entire biomedical community, because while the Ernie Beutler brand of physician-scientist is absolutely essential, it is all too rare.

\section{Kenneth Kaushansky}

Department of Medicine, University of California, San Diego, La Jolla, California, USA.E-mail: kkaushansky@ucsd.edu. 